\title{
Ultrasonography-integrated risk profile for determining the need for radiotherapy after phase I surgery
}

\author{
L. Fu, Y. Li, X. Guo, S. Chen* , G. Chen* \\ Department of ultrasound, the fourth hospital of Shijiazhuang City, Hebei Province, 050000, China
}

\section{- Original article}

\author{
*Corresponding authors: \\ Shunjue Chen, Ph.D., \\ Guihong, Chen, Ph.D., \\ E-mail: \\ prof.med.qi@gmail.com
}

Revised: January 2021

Accepted: March 2021

Int. J. Radiat. Res., October 2021; 19(4): $891-898$

DOI: $10.29242 /$ ijrr.19.4.891

\begin{abstract}
Background: To explore the efficiency of transvaginal ultrasonography in determining muscular infiltration of phase I endometrial cancer prognosis for radiotherapy. Materials and Methods: A total of 96 phase I endometrial cancer patients who were admitted between March 2018 and March 2020 were enrolled and transvaginal ultrasonography was applied for the diagnosis of muscular infiltration. Demographic variables and radiotherapy outcomes were recorded. We hypnotized that only patients with unfavorable profile of sonography receive pelvic radiotherapy. Results: Patients with muscular infiltration $>1 / 2$ had much thicker endometrium than those with muscular infiltration $\leq 1 / 2$. the sensitivity, specificity, accuracy, positive predictive value and negative predictive value of transvaginal ultrasonography in diagnosis of muscular infiltration of phase I endometrial cancer versus surgery results were $84.9 \%, 88.9 \%, 87.5 \%, 80.0 \%$ and $91.8 \%$, while the consistency and Kappa value between the transvaginal ultrasonography and pathological test was $72.2 \%$ and 0.580 . Positive Likelihood ratio of muscular infiltration more than $1 / 2$ was 2.10 (CI95\%: 1.22 to 3.61 ), meaning that approximately 10 patients of 17 patients with muscular infiltration more than $1 / 2$ would experience radiotherapy later. Negative Likelihood ratio was 0.63 (C195\%: 0.44 to 0.91 ), meaning that approximately 10 patients of 15 patients with muscular infiltration less or equal than $1 / 2$ would not experience radiotherapy later. Conclusion: Muscular infiltration of endometrial cancer in transvaginal ultrasonography could be a reliable, simple, convenient and low-cost method predicting the need for the external beam pelvic radiotherapy as an unfavorable progression in endometrial cancer.
\end{abstract}

Keywords: Transvaginal ultrasonography, endometrial cancer, phase I, muscular infiltration.

\section{INTRODUCTION}

Endometrial cancer, as a kind of endometrial epithelial malignant tumor, ranks the third in the tumor-related mortality rate in the tumors of female reproductive system, only lower than the ovarian cancer and cervical cancer (1). Peri- and post-menopause women are the high-risk population of endometrial cancer, and the clinical prevalence of endometrial cancer presents with the evident trend of increase. Current evidence has shown that early diagnosis and treatment can remarkably improve the efficacy, and appropriate, in-time treatment could increase the 5-year survival rate of patients to $80 \%$ [2]. Hence, early diagnosis and the appropriate, in-time surgical treatment are quite important to the patients. Though uterus curettage is one of the options for diagnosis of endometrial cancer, it fails to reflect the range and depth of lesions (2). Magnetic resonance imaging (MRI), though it can reflect the degree of muscular infiltration in uterus, has also been limited in the application due to the high 
expense (3). Transvaginal ultrasonography can display the size of infiltrated lesions and the Doppler indicators of the local lesions, while the depth of muscular infiltration affects directly the prognosis of endometrial cancer patients, so it is necessary to measure the depth of muscular infiltration prior to the surgery (4).

Previous studies have used molecular methods to determine the need for the radiotherapy. Based on the PORTEC-3 trial, patients with stages II and III and high-risk stage I endometrioid endometrial cancer would need radiation (5). Also, plenty studies have conducted to demonstrate the imaging characteristics of each stage of this cancer. High-risk lesions were less prone to have normal endometrialmyometrial junction, larger, and more probable to have non-uniform echogenicity compared to low-risk tumors (6). This would be easy for clinicians to decide radiotherapy initiation in high-grade cancers; but, at early stages, the decision would be doubtful.

Based on the PORTEC- 2 trial, patients with stage $1 \mathrm{C}$ grade $1 / 2$, or $1 \mathrm{~B}$ grade 3 cancer were included in study. They randomly assigned patients to groups receiving external beam radiotherapy or vaginal brachytherapy (7) and there were no definitive criteria to assign patients for these treatments. While imaging studies could be used to assess the response rate to various treatments as those are reflections of the pathological findings of the tumor; there is no such progression risk analysis based on the imaging methods even before surgery. Thus, in this study, we investigated the efficiency of transvaginal ultrasonography in diagnosis of the depth of muscular infiltration of phase I endometrial cancer and its association with the later occurrence of a radiotherapy, and the detailed information is reported as follow.

\section{MATERIALS AND METHODS}

\section{General data}

A total of 96 phase I endometrial cancer patients who were admitted to this hospital between March 2018 and March 2020 were enrolled into this study, and diagnosis was made upon the results of pathological examination, represented based on the FIGO staging (Ia/ Ib/ Ic). All patients were informed of the content of this study and agreed to participate in this study, and this study had been approved by the Ethical Board of fourth hospital of Shijiazhuang. (Ethic registration number: EC-2018-9)

Inclusion criteria was phase Ia/ Ib/ Ic endometrial cancer diagnosed by pathology findings in patients who had a surgery for the tumor removal. Patients with the severe disorders in liver or kidney, or in lactation period were excluded from this study.

A Color Doppler Ultrasonography Device (GE730, General Electric, USA) was used in this study, with the frequencies of probe in the abdomen and vagina being set between 2 and 5 $\mathrm{MHz}$ and 5.0 and $7.5 \mathrm{MHz}$. In brief, abdominal ultrasonography was performed to detect the anomalies in uterine adnexa and pelvic cavity, and patients were then required to void the bladder for vaginal ultrasonography. Coupling agent was smeared on the vaginal probe that was coated by the condom, and then the probe was placed in the vagina for continuous scanning procedures by adjusting the direction and depth of probe. Myometrial depth of infiltration was determined by experienced expert to be lower or higher than $50 \%$ infiltration (figure 1).

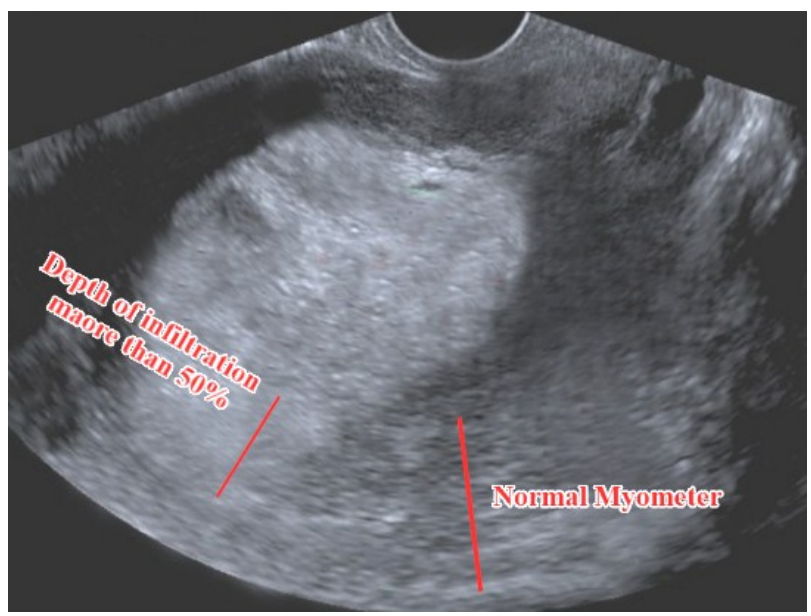

Figure 1. Myometrial depth of infiltration more than $50 \%$ Myometrial infiltration is shown in comparsion of the normal view of the myometer with no infilteration.

Int. J. Radiat. Res., Vol. 19 No. 4, October 2021 
46 Gy, with 2 Gy fractions, 5 times per week was prescribed as radiation therapy dosage for patients who were detected eligible based on the physicians decision (external beam radiotherapy machine, TSE: 6501, Hitachi Ltd. Japan).

\section{Criteria for evaluation}

Evaluation of muscular infiltration: Depth of infiltration refers to the thickness of normal muscular layer minus the depth of infiltrated lesion, and infiltration degree was presented with the ratio of infiltration depth to the thickness of normal muscular layer and categorized into three degrees - $0, \leq 1 / 2$ and $>1 / 2$.

For the criteria for evaluation of stage, phase Ia referred to the patients with complete uterine junction zone, or with complete enhanced zone of endometrium in enhancement scan, where the thickness of premenopausal endometrium $>10$ $\mathrm{mm}$ or that of postmenopausal endometrium $>5$ $\mathrm{mm}$; phase Ib referred to patients with the interruption of uterine junction zone, or with coarse surface in enhancement scan, with the signals reaching to the muscular layer $\leq 50 \%$; phase Ic referred to the patients with signals of tumor reaching to the muscular layer $>50 \%$.

We hypnotized that only patients with unfavorable profile of sonography receive pelvic radiotherapy and assessed the sonography sensitivity and specificity.

\section{Statistical analysis}

Patients' records were assessed for demographic variables and performance of an external beam pelvic radiotherapy based on the tumor characteristics. SPSS 21.0 software was utilized to perform the statistical analysis. Measurement data were shown in form of mean \pm standard deviation (SD), and the difference between two groups was validated by the $t$ test. Enumeration data were shown in form of rate (\%), and the difference between two groups was validated by the chi-square test. $P / 0.05$ suggested that the difference had statistical significance.

\section{RESULTS}

Patients aged between 34 and 59 years old, with an average age of $(48.1 \pm 2.3)$ years old. As for the distribution of clinical manifestations, there were 51 patients with postmenopausal irregular bleeding, 37 with bleeding in the reproductive age, 15 with the odor of vaginal secretions and 7 with no obvious symptoms. For the postoperative pathological examination, there were 25 patients in phase Ia, 33 in phase $\mathrm{Ib}$ and 38 in phase Ic. For the type of endometrial cancer, there were 21 patients with non-adenocarcinoma and 75 with adenocarcinoma.

\section{Comparison of the basic condition of patients} with different degrees of muscular infiltration

Patients with muscular infiltration $>1 / 2$ had much thicker endometrium than those with muscular infiltration $\leq 1 / 2$. As the histological category escalated, proportion of patients with muscular infiltration $>1 / 2$ also increased, while the proportion of those with muscular infiltration $\leq 1 / 2$ decreased evidently (all $P$ / 0.05). Patients with the lymph node metastasis had a higher proportion of muscular infiltration $>1 / 2$ than those with no lymph node metastasis, while the proportion of muscular infiltration $\leq$ $1 / 2$ was much lower (all $P / 0.05$ ). For patients with varying menopausal condition and pathological types, the proportions of patients with muscular infiltration $>1 / 2$ or $\leq 1 / 2$ showed no significant difference $(P=0.05$; table 1).

\section{Analysis of the parameters of transvaginal ultrasonography in evaluation of the muscular infiltration in phase I endometrial cancer}

The sensitivity, specificity, accuracy, positive predictive value and negative predictive value of transvaginal ultrasonography in diagnosis of muscular infiltration of phase I endometrial cancer were $84.9 \%(28 / 33), 88.9 \%$ (56/63), $87.5 \%(84 / 96), 80.0 \%(28 / 35)$ and $91.8 \%$ $(56 / 61)$ (table 2). 
Table 1. Comparison of the basic condition of patients with different degrees of muscular infiltration [n (\%)].

\begin{tabular}{|c|c|c|c|c|c|}
\hline \multicolumn{2}{|c|}{ Clinical features } & $\begin{array}{c}\text { Muscular infiltration } \\
\qquad>1 / 2(n=33)\end{array}$ & $\begin{array}{l}\text { Muscular infiltration } \\
\leq 1 / 2(n=63)\end{array}$ & $t / c^{2}$ & $\boldsymbol{P}$ \\
\hline \multicolumn{2}{|c|}{ Thickness of endometrium (mm, mean \pm SD) } & $31.1 \pm 5.3$ & $20.2 \pm 3.4$ & 3.91 & $<0.05$ \\
\hline \multirow{2}{*}{$\begin{array}{l}\text { Condition of } \\
\text { menopause }\end{array}$} & Menopausal & $23(41.1)$ & $33(58.9)$ & \multirow{2}{*}{1.48} & \multirow{2}{*}{$>0.05$} \\
\hline & Non-menopausal & $11(27.5)$ & $29(72.5)$ & & \\
\hline \multirow{2}{*}{ Pathological types } & Adenocarcinoma & $24(32.0)$ & $51(68.0)$ & \multirow{2}{*}{0.76} & \multirow{2}{*}{$>0.05$} \\
\hline & Non-adenocarcinoma & $9(42.9)$ & $12(57.1)$ & & \\
\hline \multirow{3}{*}{ Histological grades } & G1 & $8(14.0)$ & $49(86.0)$ & \multirow{3}{*}{21.68} & \multirow{3}{*}{$<0.05$} \\
\hline & G2 & 14(60.9) & $9(39.1)$ & & \\
\hline & G3 & $12(75.0)$ & $4(25.0)$ & & \\
\hline \multirow{2}{*}{$\begin{array}{l}\text { Lymph node } \\
\text { metastasis }\end{array}$} & Yes & $11(73.3)$ & $4(26.7)$ & \multirow{2}{*}{8.28} & \multirow{2}{*}{$<0.05$} \\
\hline & No & $22(27.2)$ & $59(72.8)$ & & \\
\hline
\end{tabular}

Table 2. Analysis of the parameters of transvaginal ultrasonography in evaluation of the muscular infiltration in phase I endometrial cancer $(n)$.

\begin{tabular}{|c|c|c|c|}
\hline \multirow{2}{*}{$\begin{array}{c}\text { Transvaginal } \\
\text { ultrasonography }\end{array}$} & \multicolumn{3}{|c|}{ Muscular infiltration } \\
\cline { 2 - 4 } & $>1 / 2$ & $\leq 1 / 2$ & Total \\
\hline$>\mathbf{1 / 2}$ & 28 & 7 & 35 \\
\hline $\mathbf{\leq 1 / 2}$ & 5 & 56 & 61 \\
\hline Total & 33 & 63 & 96 \\
\hline
\end{tabular}

Analysis of the consistency in diagnosis of phase I endometrial cancer with transvaginal ultrasonography and pathological

According to the analysis, the consistency and Kappa value between the transvaginal ultrasonography and pathological test was $72.2 \%$ and 0.580 (table 3 ).

\section{Radiotherapy receiving outcome}

Considering the Muscular infiltration more than $1 / 2$ positive for Transvaginal ultrasonography and Muscular infiltration $\leq 1 / 2$ as negative, the Likelihood of the need for external beam pelvic radiotherapy was assessed. Based on the results, 21 patients who had Muscular infiltration $>1 / 2$, and 19 patients with muscular infiltration $\leq 1 / 2$, received external beam pelvic radiotherapy (table 4 ).

Based on the table 5 and figure 2, Positive Likelihood ratio was 2.10 (CI95\%: 1.22 to 3.61). Negative Likelihood ratio was 0.63 (CI95\%: 0.44 to 0.91 ).
Table 3. Analysis of the consistency in diagnosis of phase I endometrial cancer with transvaginal ultrasonography and pathological (n).

\begin{tabular}{|c|c|c|c|c|}
\hline Transvaginal & \multicolumn{4}{|c|}{ Pathological stages } \\
\cline { 2 - 5 } ultrasonography & la & Ib & Ic & Total \\
\hline la* $^{*}$ & 17 & 8 & 0 & 25 \\
\hline Ib* $^{*}$ & 5 & 24 & 4 & 33 \\
\hline Ic* $^{*}$ & 3 & 7 & 28 & 38 \\
\hline Total & 25 & 39 & 32 & 96 \\
\hline
\end{tabular}

Note: according to FIGO staging la shows that tumor limited to one ovary; capsule intact, no tumor on ovarian surface; no malignant cells in ascites or peritoneal washings, lb shows that Tumor limited to both ovaries; capsules intact, no tumor on ovarian surface; no malignant cells in ascites or peritoneal washings and Ic shows that tumor limited to one or both ovaries with any of the subcategories below.

Table 4. Positive and negative transvaginal ultrasonography vs. Radiotherapy occurrence.

\begin{tabular}{|c|c|c|c|}
\hline \multirow{2}{*}{$\begin{array}{c}\text { Transvaginal } \\
\text { ultrasonography } \\
\text { for Muscular infiltration }\end{array}$} & \multicolumn{3}{|c|}{ External beam pelvic radiotherapy } \\
\hline & yes & no & Total \\
\hline$>1 / 2$ & 21 & 14 & 35 \\
\hline$\leq 1 / 2$ & 19 & 42 & 61 \\
\hline Total & 40 & 56 & 96 \\
\hline
\end{tabular}

Table 5. Likelihood ratio of test.

\begin{tabular}{|c|c|c|}
\hline & Value & $\mathbf{9 5 \%} \mathrm{Cl}$ \\
\hline Positive Likelihood ratio & 2.10 & 1.22 to 3.61 \\
\hline $\begin{array}{c}\text { Posterior probability of } \\
\text { Positive test (odds) }\end{array}$ & $60 \%(1.5)$ & $47 \%$ to $72 \%$ \\
\hline Negative Likelihood ratio & 0.63 & 0.44 to 0.91 \\
\hline $\begin{array}{c}\text { Posterior probability of } \\
\text { Negative test (odds): }\end{array}$ & $31 \%(0.5)$ & $24 \%$ to $39 \%$ \\
\hline
\end{tabular}




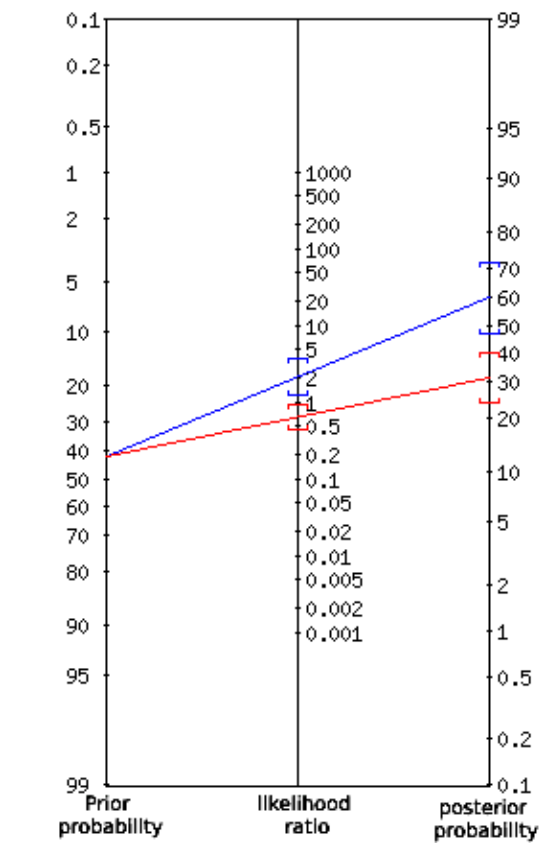

Figure 2. Nomogram of the Likelihood ratio.

In this nomogram, the left, middle, and right axes represent the pre-test probability, likelihood, and post-test probability, respectively. To use this nomogram, the pre-test and likelihood probabilities in the left and middle axes get marked (left side). Then a straight line from the mark on the left axis to the mark on the middle axis and extended to the line to the right axis is drawn. The location of the intersection of this line and the axis on the right will indicate the probability after the test. Meaning that approximately 10 patients of 15 patients with muscular infiltration less or equal than $1 / 2$ would not experience radiotherapy later and approximately 10 patients of 17 patients with muscular infiltration more than $1 / 2$ would experience radiotherapy later.

\section{DISCUSSION}

While some scholars believe that in the early stage of endometrial cancer, patients present with the normal result in ultrasonography, with no evident enlargement in uterus, so ultrasonography is only suitable for patients with tumor invasion into the muscle layer (8-10). Nearly half of the endometrial cancer patients are diagnosed in the postmenopausal period, with the major clinical manifestation of vaginal bleeding. As the prevalence of endometrial cancer increases, a method that can reflect the phase of endometrial cancer is of great significance for the rational evaluation of prognosis and appropriate treatment (11). Especially for patients with phase I endometrial cancer, accurate evaluation of the infiltration depth of tumor into the muscle is key to the selection of surgical methods.

As reported, no muscular infiltration and the specified infiltration depth into the muscular layer should be taken as the criteria for evaluating the phase, where patients in phase Ia have tumors in the endometrium, with no infiltration into the muscular layer, patients in phase Ib have tumors that infiltrate into the superficial layer of muscle $(\leq 50 \%)$, and those in phase Ic have tumor infiltrating into the deep layer of muscle (>50\%) (12). Moreover, the varying degrees of muscular infiltration in the endometrial cancer patients affect the efficacy and the risk of lymph node metastasis, where patients in phase Ia usually are free from the lymph node metastasis, patients in phase Ib have incidence rate of $30 \%$ in lymph node metastasis and those in phase Ic have incidence rate of over $50 \%$ in lymph node metastasis (13). Besides, patients with the muscular infiltration have a significant increase in the recurrence rate even though they receive the appropriate therapy. Therefore, early diagnosis and appropriate treatment could maximize the survival time of patients.

Our study showed that approximately 10 patients of 15 patients with muscular infiltration less or equal than $1 / 2$ would not experience radiotherapy later after the surgery. Also, 10 patients of 17 patients with muscular infiltration more than $1 / 2$ would experience radiotherapy later. Previous studies had attempts to predict the need for radiotherapy based on the molecular characteristics of the tumor. Unfavorable profile of endometrial cancer which needs external beam pelvic radiotherapy was defined by van den et al. (14). Molecular-integrated risk profile was suggested by Wortman et al. also confirmed the worse 
condition of the patient and the need for the radiotherapy. This was also confirmed in our study; while we used sonography-integrated risk profile (15).

MRI is believed to be an efficient tool in evaluating the muscular infiltration, but due to the high expense, a great number of patients have to give up this choice. Transvaginal ultrasonography possesses many advantages, including the simple, convenient and repeatable operation, high resolution, no invasion, low cost and immunization from the effect of obesity and intestinal gas (16). Besides, the multi-frequency probe can display the ovary, uterus and the adjacent tissues of patients clearly, while color ultrasonography can reflect the changes in the hemodynamics of patients, in which the resistance of blood flow can be demonstrated by the change in the frequency spectrum. Thus, color doppler ultrasonography in combination with the uterine curettage is the major way for diagnosis of endometrial cancer (17).

Transvaginal ultrasonography is a kind of non-invasive technique, and the high-resolution probe can display the structure of uterus, identify the muscular layers and endometrium precisely and evaluate the muscular infiltration of patients, thus providing solid evidence for the staging of endometrial cancer. In the diagnosis of phase I endometrial cancer, transvaginal ultrasonography is the first choice for preoperative examination (18-19).

In this study, we found that patients with muscular infiltration $>1 / 2$ had much thicker endometrium than those with muscular infiltration $\leq 1 / 2$. As the histological category escalated, proportion of patients with muscular infiltration $>1 / 2$ also increased, while the proportion of those with muscular infiltration $\leq 1 / 2$ decreased evidently (all $P / 0.05$ ). Patients with the lymph node metastasis had a higher proportion of muscular infiltration $>1 / 2$ than those with no lymph node metastasis, while the proportion of muscular infiltration $\leq 1 / 2$ was much lower (all $P / 0.05$ ).

For patients with varying menopausal condition and pathological types, the proportions of patients with muscular infiltration $>1 / 2$ or $\leq 1 / 2$ showed no significant difference $(P=0.05)$. Besides, the sensitivity, specificity, accuracy, positive predictive value and negative predictive value of transvaginal ultrasonography in diagnosis of muscular infiltration of phase I endometrial cancer were $84.9 \%, 88.9 \%, 87.5 \%, 80.0 \%$ and $91.8 \%$, while the consistency and Kappa value between the transvaginal ultrasonography and pathological test was $72.2 \%$ and 0.580 . These findings are consistent with the previous results (20).

In another study, Yela et al. showed that in postmenopausal patients, ultrasonography has a sensitivity of $99.0 \%$, specificity of $19.0 \%$, PPV of $96.1 \%$, NPV of $50.0 \%$, and accuracy of $95.3 \%$, and in contrast, hysteroscopy has a sensitivity of $96.7 \%$, specificity of $86.9 \%$, PPV of $99.2 \%$, NPV of $58.8 \%$, and accuracy of $96.2 \%$ (21). However, in the present study, we examined bedside sensitivity.

Mohammad et al. conducted a study comparing the diagnostic power and sensitivity of three-dimensional and hysteroscopic transvaginal ultrasound in the diagnosis of intrauterine lesions. In their study The reliability, precision, positive predictive value, negative predictive values and cumulative overall accuracy of Three-dimensional transvaginal ultrasound for total irregular outcomes was $89.13 \%, 100 \%, 100 \%, 44.44 \%$ and $90 \%$, respectively, compared with the use of hysteroscopy (22). In line with the results of our study, the results of Mohammad et al. study also show the high sensitivity of ultrasound in the diagnosis of intrauterine lesions.

In another study 255 patients with irregular uterine bleeding by ultrasound, sonohysterography and operative hysteroscopy were examined by El Tabbakh et al. Histological examination revealed endometrial hyperplasia in 70 patients where sensitivity, specificity, positive predictive value, negative predictive value and diagnostic accuracy were 77\%, 94.6\%, $84.4 \%, 91.6 \%$ and $89.8 \%$ for transvaginal ultrasound and 95.7\%, 96.8\%, 91.8\%, 98.35\% and $96.5 \%$ for sonohysterography ND $75.7 \%$, $97.3 \%, 91.4 \%, 91.45,91.4 \%$ for hysteroscopy $(23)$.

Int. J. Radiat. Res., Vol. 19 No. 4, October 2021 


\section{CONCLUSION}

Overall, Muscular infiltration of phase I endometrial cancer in transvaginal ultrasonography could be assumed as an indicator of radiotherapy requirement after surgery in endometrial cancer research. Further studies in clinical trials would help to determine the benefits of taking muscular infiltration into account as an indication of radiotherapy. But, this approach for predicting the need for the external beam pelvic radiotherapy as an unfavorable progression in endometrial cancer is simple, convenient and low-cost.

\section{Conflicts of interest: Declared none.}

\section{REFERENCES}

1. Lu HX, Song JX, Li Y (2018) Diagnostic value of ultrasound elastography and magnetic resonance diffusion imaging in cervical cancer. J of Zhengzhou Uni: Medical Edition, 53(4): 492-496.

2. Kong XC and Song TF (2018) Application of imaging examination in the diagnosis of pregnancy complicated with gynecologic tumor. Chin J of Practic Gyneco and Obstetrics, 34(10): 1098-1102.

3. Li XX, Huang QQ, Wen BY (2018) The value of miRNA combined with transvaginal color Doppler ultrasound in the diagnosis of cervical cancer. Mod Prevent Med, 45(17): 3248-3252.

4. Mao YJ, Zheng ZJ, Xu J (2017) Evaluation of neoadjuvant chemotherapy for cervical cancer by three-dimensional power Doppler ultrasound. Chin J of Ultra Med, 33(7): 616 -620 .

5. de Boer SM, Powell ME, Mileshkin L, Katsaros $D$, Bessette $P$, Haie-Meder C, Ottevanger PB, Ledermann JA, Khaw $P$, D'Amico R, Fyles A (2019) Adjuvant chemoradiotherapy versus radiotherapy alone in women with high-risk endometrial cancer (PORTEC-3): patterns of recurrence and post-hoc survival analysis of a randomised phase 3 trial. The Lancet Onco, 20(9): 1273-1285.

6. Epstein E, Fischerova D, Valentin L, Testa AC, Franchi D, Sladkevicius $P$, Frühauf, F, Lindqvist PG, Mascilini F, Fruscio R, Haak LA (2018) Ultrasound characteristics of endometrial cancer as defined by International Endometrial Tumor Analysis (IETA) consensus nomenclature: prospective multicenter study. Ultrasound in Obste \& Gyneco, 51(6): 818828.

7. Nout RA, Smit VT, Putter H, Juergenliemk-Schulz IM, Jobsen JJ, Lutgens LC, van der Steen-Banasik EM, Mens
JW, Slot A, Kroese MS, van Bunningen BN (2010) Vaginal brachytherapy versus pelvic external beam radiotherapy for patients with endometrial cancer of high-intermediate risk (PORTEC-2): an open-label, non-inferiority, randomised trial. The Lancet, 375(9717): 816-23.

8. Exner M, Kuehn A, Stumpp P, Hoeckel M, Horn LC, Kahn T, Brandmaier P (2016) Value of diffusion weighted MRI in diagnosis of uterine cervical cancer: a prospective study evaluating the benefits of DWI comparedto conventional MR sequences in a $3 \mathrm{~T}$ environment. Acta Radiol, 57(7): $869-869$.

9. Wang YT, Li YC, Yin LL, Pu H (2016) Can diffusion-weighted magnetic reso-nance imaging predict survival in patients with cervical cancer: a meta - analysis. Eur J Radiol, 85(12): $2174-2174$.

10. Li H, Chang SF, Deng LB (2018) Study on the accuracy of transvaginal two-dimensional ultrasonography in cervical morphology and volume. Chin J of Practic Gyneco and Obstetrics, 34(8): 928-930.

11. Jiang X, Niu K, Hu XJ (2017) Evaluation of the efficacy of radiotherapy and chemotherapy for cervical cancer by conventional intracavitary ultrasound combined with realtime shear wave elastography. Chin J of Ultrasound Med, 33(7): 660-662.

12. Zhang $Y$ and Luo YH (2017) Application of imaging in the diagnosis of cervical cancer. Radio Practice, 32(4): 336340.

13. Li JP, Chen DF, Wang SC (2016) Quantitative evaluation of cervical cancer and precancerous lesions by transvaginal ultra-high speed shear wave elastography. Chin J of Imag Techno, 32(11): 1706-1709.

14. van den Heerik AS, Horeweg N, de Boer SM, Bosse T, Creutzberg CL (2020) Adjuvant therapy for endometrial cancer in the era of molecular classification: radiotherapy, chemoradiation and novel targets for therapy. Int $J$ of Gynec Cancer, 18: ijgc-2020.

15. Wortman BG, Bosse T, Nout RA, Lutgens LC, van der Steen -Banasik EM, Westerveld $H$, van den Berg $H$, Slot A, De Winter KA, Verhoeven-Adema KW, Smit VT (2018) Molecular-integrated risk profile to determine adjuvant radiotherapy in endometrial cancer: evaluation of the pilot phase of the PORTEC-4a trial. Gyneco Onco, 151(1): 69-75.

16. Dodampahala SH, Jayakody SN, Gunathilake WC, Rahubaddha AN, Dodampahala SK (2016) Transvaginal color Doppler in the assessment of cervical carcinoma and precancer: evidence from a case control study using colour Doppler ultrasonography pulsatility index of uterine vasculature. Adv Reprod Sci, 4(3): $93-93$.

17. Ma XI, Li QI, Wang JL, Shao J, Zhu YC, Ding W, Zhang HS, Wang HY, Shen JK (2017) Comparison of elastography based on transvaginal ultrasound and MRI in assessing parametrial invasion of cervical cancer. Clin Hemorheol Microcirc, 66(1): $27-35$.

18. Li XH, Li JP, Xu H (2017) Value of transvaginal ultra-high speed shear wave elastography in the diagnosis of cervical cancer and precancerous lesions. Chin J of General Prac- 
tice, 20(18): 2285-2288.

19. Yin PP, Li JP, Wang SC (2018) Real time transvaginal ultrasound elastography in the diagnosis of cervical cancer: a meta-analysis. Chin Med Imag Techno, 34(11): 1681-1685.

20. Alcázar JL, Galván R, Albela S, Martinez S, Pahisa J, Jurado M, López-García G (2009) Assessing myometrial infiltration by endometrial cancer: uterine virtual navigation with three-dimensional US. Radiology, 250(3):776-83.

21. Yela DA, Pini PH, Benetti-Pinto CL (2018) Comparison of endometrial assessment by transvaginal ultrasonography and hysteroscopy. Int J of Gynec \& Obstetrics, 143(1):32-6.

22. Mohammad FA, Abdal Fattah AT, Abd-Elrahman AM
(2018) Comparative study between three-dimensional transvaginal ultrasonography and hysteroscopy in the diagnosis of uterine cavity abnormalities. The Egypt $J$ of Hospital Med, 73(8): 7350-7.

23. El-Tabbakh MN and Slamka P (2011) Transvaginal ultrasonography, sonohysterography, hysterosalpingography and operative hysteroscopy. Available at: http:// www. obgyn. Net / obgyn- ultrasound/ transvaginal- ultrasonographysonohysterographyhysterosalpingography-andoperativehysteroscopy. 